\title{
BMJ Open Cohort profile: improving treatment of HIV-infected Ethiopian children through better detection of treatment failure in southern Ethiopia
}

\author{
Birkneh Tilahun Tadesse, ${ }^{1}$ Byron Alexander Foster, ${ }^{2}$ Degu Jerene, ${ }^{3}$ Andrea Ruff ${ }^{4}$
}

To cite: Tadesse BT, Foster BA, Jerene D, et al. Cohort profile: improving treatment of HIV-infected Ethiopian children through better detection of treatment failure in southern Ethiopia. BMJ Open 2017;7:e013528. doi:10.1136/bmjopen-2016013528

- Prepublication history and additional material is available. To view please visit the journal (http://dx.doi.org/ 10.1136/bmjopen-2016013528).

Received 19 July 2016 Revised 27 October 2016 Accepted 22 December 2016

\section{CrossMark}

${ }^{1}$ Department of Pediatrics, Hawassa University, Hawassa, Ethiopia ${ }^{2}$ University of Texas Health Science Center at San Antonio, San Antonio, Texas, USA

${ }^{3}$ Management Sciences for Health, Health Programs Group, Addis Ababa, Ethiopia ${ }^{4} J o h n s$ Hopkins University Bloomberg School of Public Health, Baltimore,

Maryland, USA

Correspondence to Dr Birkneh Tilahun Tadesse; birknehtilahun@gmail.com

\section{ABSTRACT}

Purpose: The Ethiopian Paediatric HIV Cohort (EPHIC) was established to identify clinical and laboratory predictors of virological treatment failure to ultimately develop a clinical-immunological prediction rule with area under the curve of $>0.80$ for detecting first-line antiretroviral therapy failure (ARTF). It will also assess the performance of the current WHO guidelines for detection of first-line ARTF in children.

Participants: Using a prospective cohort design, HIV-infected children and adolescents below the age of 18 years are followed every 6 months with a set of clinical and laboratory parameters at 6 hospitals in southern Ethiopia. For inclusion in the cohort, children should be on or are initiating first-line antiretroviral therapy (ART) and are not on second-line ART. Virological treatment failure is taken as the gold standard for the diagnosis of treatment failure.

Findings to date: From October 2015 through April 2016, 628 children have been enrolled from 6 different HIV treatment centres across southern Ethiopia. The mean age at enrolment was 11.1 years and $47.6 \%$ were girls. Many of the children $(88.6 \%)$ were at WHO Clinical stage 1 at time of enrolment. At enrolment, the mean duration on first-line ART was 45 months. Substitution of ART drugs was performed to nearly half $(42.6 \%)$ of the cohort. Adherence as assessed with the Visual Analogue Scale was high (mean, 94.4\%; $\mathrm{SD}=11.9$ ). The median CD4 count of the cohort at enrolment was 741 with $3.1 \%$ having a value consistent with ARTF.

Future plans: Regular data uploads from the 6 hospitals in southern Ethiopia enable this cohort to be followed prospectively. The cohort will be completed in September 2017. The successful completion of this study will allow for better targeting of viral-load testing to those at highest risk in resource-poor settings and provide clinicians and policymakers with a practical prediction rule.

Ethics approval: SNNPR Regional Health Bureau Institutional Review Board.

\section{INTRODUCTION}

As of 2015, 1.8 million children $<15$ years of age were living with HIV among which 1.5

\section{Strengths and limitations of this study}

- The study will be the first paediatric scoring system for earlier detection of first-line antiretroviral treatment failure in resource-limited settings where viral load facility is limited or not accessible.

- The prediction rule will be an important tool for targeting viral load measurement in settings where there is limited viral load facility and could be an alternative for earlier detection of treatment failure in settings where viral load is not accessible.

- Although different mechanisms have been devised, non-adherence and loss to follow-up could be challenges in the study.

million were living in sub-Saharan Africa. ${ }^{1}$ While the use of antiretroviral therapy (ART) has improved clinical and immunological outcomes of children, ${ }^{2}{ }^{3}$ issues of drug resistance and treatment failure have emerged.

Treatment failure is considered when a child who has taken ART for at least 6 months presents with clinical, immunological or virological evidence of unsuccessful treatment. ${ }^{4}$ Time to treatment failure from initiation of ART appears to depend on several factors, including genetics, adherence, previous ART exposure, transmitted HIV resistance and age in children; and it can occur from 6 months to many years. ${ }^{5-7}$ In Ethiopia, immunological treatment failure has been reported to be $4-17.5 \%$ among adults, ${ }^{8-10}$ and in children who are on firstline ART, 5.9\% clinical and 6.7\% immunological treatment failure were reported. ${ }^{5}$ In the absence of viral load monitoring treatment failure is diagnosed using immunological and clinical parameters following the WHO guidelines. ${ }^{4}$

Viral load testing is the gold standard for monitoring ART. ${ }^{11}$ However, this test is expensive and technically demanding, ${ }^{12}$ 
functionally prohibiting its use in resource-constrained settings. ${ }^{13} 14$ In developing countries, viral load determination is not available as a diagnostic tool for the detection of treatment failure in children. As part of the 90-90-90 plan, the current Ethiopian strategy for the testing, treatment and follow-up of virological suppression, efforts are being made to make viral load testing available in regional laboratories. ${ }^{15}$ However, in remote areas and in settings where viral load determination is logistically difficult and not easily accessible, the WHO 2016 guidelines emphasise targeting viral load testing using clinical and immunological parameters. ${ }^{16}$ Therefore, in low resources settings, developing a clinical prediction rule which requires the use of fewer resources is important to prioritise viral load testing and target the available limited viral load facility.

Analysing the clinical and immunological predictors used in the WHO criteria for detection of first-line ART failure (ARTF) in children and sorting them in their order of strength based on likelihood ratios (LR) to predict treatment failure may lead to a more accurate prediction rule for detection of first-line ARTF. Predictors in the WHO criteria identified as strong will be used to build the new prediction rule. In the current study, using a thorough analysis of the possible clinical and immunological predictors, we will be able to identify the strongest parameters that predict first-line ARTF in children. Ultimately, developing an accurate prediction rule for first-line ARTF will minimise the need for viral load determination. Consequently, this will decrease the cost and delay incurred by viral load determination and will decrease the duration children stay on a failing regimen and the resulting drug resistance, potentially decreasing the number of AIDS deaths among HIV-infected children.

The objectives of this prospective cohort study are therefore threefold: (1) determine the performance of the WHO clinical criteria for detection of first-line ARTF in HIV-infected Ethiopian children; (2) identify important clinical and immunological predictors of first-line ARTF among HIV-infected Ethiopian children and (3) develop a practical, acceptable and accurate clinical prediction rule to improve detection of first-line ARTF among HIV-infected Ethiopian children. In this paper, we describe the basic design, recruitment and characteristics of the cohort.

\section{COHORT DESCRIPTION}

\section{Study design and inclusion criteria}

This prospective cohort study includes children under 18 years with a diagnosis of HIV based on WHO guidelines, ${ }^{4}{ }^{17}$ who are eligible for or are already on first-line ART. On the basis of the Ethiopian guideline for ART care in children, all children infected with HIV are initiated on ART. Children who discontinue follow-up before 6 months on ART are on second-line ART or with ARTF at enrolment are excluded.
The study is being carried out in the Southern Nations Nationalities and Peoples Region (SNNPR) of Ethiopia, where in 2012, there were 27200 children below 14 years of age with HIV, 12258 of whom needed ART. ${ }^{18}$ In SNNPR, paediatric ART services are provided in 22 hospitals. We enrolled participants in the first 6 months of the study at the following six established paediatric ART centres with the highest patient volume in SNNPR: Hawassa University; Yirgalem; Arba Minch; Sodo; Adare and Hosana hospitals.

\section{Baseline data}

On enrolment, through medical chart review and use of structured questionnaires, we collected the demographics (age, gender, race) of the child; clinical data (age at diagnosis and ART initiation), history of prevention of mother to child transmission (PMTCT) exposure, nutritional status, WHO clinical stage, mode of transmission, current or past tuberculosis infection and current ART regimen and laboratory parameters (leucocyte count, thrombocyte count, haemoglobin, CD4 count/per cent, viral load).

\section{Clinical data}

Patients will be followed every 6 months (when they come for their routine follow-up) for development of new symptoms using a structured symptom checklist. Growth parameters will be assessed using the WHO growth standards, ${ }^{19}$ along with assessment of age-appropriate developmental milestones. ${ }^{4}$ Body mass index (BMI) z-score and other growth parameters are calculated using Epi Info (CDC, USA 2016). Adherence will be assessed using a Visual Analogue Scale (VAS) by trained nurse data collectors, ${ }^{20}$ and self-report on missed doses in the previous week and month.

\section{Immunological data}

Complete blood count (CBC) and CD4 count will be checked every 6 months during their routine follow-up; CD4 percentage and per cent change in CD4 count from previous record will be determined.

\section{Virological data}

We will determine viral load at baseline and every 6 months for 2 years. Viral load testing will be carried out using Abbott RealTime HIV-1 m2000rt m2000sp machine with calibration performed before running every batch of samples. If the child has any acute illness, viral load determination will be deferred until the infection resolves. ${ }^{4}$

\section{Patient care}

During visits, decision to switch ART or other treatments will be made by the clinical staff and will not be directed by the study. Viral load testing results from the study will be reported to the treating clinician. Ethiopia is currently rolling out viral load testing to be performed yearly across the country. 


\section{Assessing performance of WHO guidelines}

In the course of follow-up, patients will be followed for any evidence of treatment failure based on the WHO 2013 guidelines. ${ }^{4}$ The proportion of children who are correctly identified by WHO guidelines will be checked against viral load.

\section{WHO 2013 definition of ARTF}

Clinical: New or recurrent clinical events indicating advanced/severe immunodeficiency (WHO clinical stage 3 and 4 clinical condition with exception of tuberculosis) after 6 months of effective treatment. ${ }^{4}$ Immunological: Children younger than 5 years: persistent CD4 levels below 200 cells $/ \mathrm{mm}^{3}$ or $<10 \%$; and, older than 5 years: persistent CD4 levels below 100 cells $/ \mathrm{mm}^{3}$. Virological (Gold Standard): HIV RNA of more than 1000 copies/mL on at least two measurements after 24 weeks of treatment. When the viral load is more than 1000 copies $/ \mathrm{mL}$, a repeat viral load test will be performed 3 months after adherence support is given.

\section{Derivation of scoring system}

In the cohort, participants will be followed until virological failure occurs or at least until 24 months after initiation of first-line ART. We will identify cases of HIV-infected children who develop ARTF and age, duration of ART and sex-matched controls among those who did not develop ARTF for at least 2 years after treatment started. Associations with clinical and laboratory predictors of ARTF will be performed using these two groups. Parameters will include CD4 at baseline, CD4 change at 6 month, haemoglobin level, persistent thrombocytopenia, nutritional status at baseline, WHO staging at baseline, failure to thrive/growth faltering, PMTCT history, adherence using VAS, duration on ART, mode of HIV infection and chronic gastroenteritis. Two by two tables will be created for each predictor. Bivariate analysis using $\chi^{2}$ test and estimation of LR will be performed.

The Spiegelhalter method will be used to build a prediction rule. ${ }^{21}$ A prediction rule will be developed using the composite of predictors with adjusted LR of more than 1.5 or $<0.6$. Log natural transformation will be performed to the adjusted LR and will be used to build final score of predictors. A receiver operating characteristic (ROC) curve will be created; cut-off point on the ROC curve will be determined using the Zweig and Campbell formula as adapted by Lynen et $a l^{22}$ in Cambodia; assuming the value of false positives to be the same as false negatives. A simplified diagnostic algorithm will be developed. Performance of the new prediction rule in detecting treatment failure will be compared with the WHO guidelines.

\section{Sample size}

To test the hypothesis that WHO criteria for detection of first-line ARTF have sensitivity of $10 \%$ and specificity of $40 \%{ }^{23}$ with precision of $5 \%$; in a population with ARTF prevalence of $15 \%,{ }^{5}$ a minimum of 960 participants will be required. Identification of important predictors to set up a prediction rule will require multiple sample size calculations for presumed predictors. The largest sample size is derived from history of maternal ART exposure. Considering prevalence of children who have ARTF to be $15 \%{ }^{5}$ with allocation ratio of cases to controls to be 0.2 ; and taking proportion of prior ART exposure in controls to be $11.5 \%$ based on a study in Mozambique, ${ }^{5624}$ at $80 \%$ power and $5 \%$ level of significance, a sample size of 705 controls and 141 cases is needed to prove that the OR for failing first-line ART is 2. To account for loss to follow-up, we will include an additional $10 \%$, therefore a minimum of 1056 children will be enrolled in this study. Matching controls by age and duration of ART will help address the heterogeneity of this population.

\section{Data quality assurance}

Data will be entered electronically, which can be accessed at the hub institute through Redcap. There will be paper back up at each site. A one-day, intensive training was conducted with HIV clinic nurses on data abstraction, data and sample collection. The training included an overview of the study purpose and design, training in the electronic data entry system, a detailed walk-through of each form and practice sessions using sample charts for data abstraction and entry with direct feedback provided to each participant. Supervision visits will be made every 3 months to check the quality of clinical and immunological data collection. Refresher trainings for the data collectors and supervisors will be given based on the observed deficiencies. Viral load determination is performed following standard operating procedures and during each run three controls are carried out to control quality.

Historical data on prior clinical staging, maternal ART prophylaxis and the child's age at diagnosis, previous treatment regimens and any changes will be abstracted from the child's chart by the nurses on the research team at the time of enrolment.

\section{Ethics and consent}

Only volunteers are included in the study. Each participant receives written information about the study in their own local language, and it is ensured that the consent is signed and dated by the parent or caregiver and the physician or ART nurse. Children older than 12 years can also give assent. Participants will be given compensation for travel and lunch for visitations for the purpose of the study, and all the costs related to the laboratory tests for the study are covered by the study. Ethical approvals have been obtained from the IRB of the regional health bureau and Hawassa University, College of Medicine and Health Sciences. Privacy is assured by removing patient identifiers, including names and specific addresses. 


\section{FINDINGS TO DATE}

From October 2015 through April 2016, 628 children have been enrolled from six different HIV treatment centres across southern Ethiopia.

\section{Demographic characteristics}

The mean age of the children at enrolment was 11.1 years, and $47.6 \%$ of the children were girls (table 1 ). The median reported income was about US $\$ 1$ per day (US $\$ 33 /$ month) with $91.2 \%$ of caregivers reporting having a job with a wide range of reported occupations (table 1). Of mothers in the cohort, $27.1 \%$ had no formal education, and $14.7 \%$ of fathers had no formal education. Only $48.0 \%$ of the children in this cohort had both parents alive, and $71.1 \%$ of the caregivers providing data were HIV positive. In contrast, a minority of caregivers reported that the child had living siblings with HIV $(15.0 \%)$.

\section{Clinical characteristics}

Overall, $81.4 \%$ of children were adequately nourished at enrolment by BMI z-score using WHO criteria (table 2). The vast majority of children were at WHO Clinical stage 1 at the time of enrolment into the cohort $(88.6 \%)$, though only $20.6 \%$ were at stage 1 at the time of initiation of ART (table 2) with a mean of 45 months between initiation of ART and enrolment in this study. Almost no children $(0.8 \%)$ had a viral load test performed at initiation of their ART. Nearly half $(42.6 \%)$ of the children had some substitution of their ART drugs with $70.3 \%$ of those being due to a national guideline change where replacement of zidovudine or tenofovir for stavudine-based regimens was performed for all patients in Ethiopia.

About one-quarter (24.7\%) of children had ever been diagnosed with tuberculosis. Of those, $91.2 \%$ had a diagnosis of pulmonary tuberculosis. Only $45.6 \%$ of children had their HIV status disclosed to them as per the caregiver, and $23.9 \%$ of the caregivers reported being worried about stigma related to HIV (table 2).

Adherence as assessed with the VAS was high with 94.4\% ( $\mathrm{SD}=11.9$ ) reporting adherence when asked over the past month, how often the child had taken their HIV medication. However, when asked how many doses over the past week the child had missed, $15.1 \%$ of caregivers reported missing at least 1 dose.

\section{Laboratory characteristics}

The enrolled children had a median CD4 count of 741 at the time of enrolment with $3.1 \%$ having a value consistent with ARTF using immunological criteria at the time of enrolment assessment (table 3). Other laboratory parameters are shown in table 3 .

\section{DISCUSSION}

Currently, the use of viral load as a follow-up tool for the diagnosis of treatment failure is emphasised by the
Table 1 Baseline demographic characteristics of enrolled patients across all six sites*

Age at enrolment, years, mean (SD)

$11.1(3.6)$

Sex, \% female (n)

Age at diagnosis, years, mean (SD)

$47.6 \%(296)$

Family size, mean (SD)

$4.7(3.7)$

Monthly income in US dollars, median (IQR)

$5.5(19.9)$

Ethnicity, \% of reporting $(n)$

Amhara

$33(19-70)$

Oromo

$19.2 \%(120)$

Sidama

$12.5 \%(78)$

$12.9 \%(81)$

Wolaita

$24.6 \%(154)$

Tigray

$1.9 \%(12)$

Gurage

$4.5 \%(28)$

Silte

$0.8 \%(5)$

Gamo

Other

Occupation of caregivers, \% of reporting ( $n$ )

Farmers

$6.9 \%(43)$

$16.8 \%(105)$

Government employees

Merchants

Daily labourers

Other

Jobless

Mother's education, \% of reporting ( $n$ )

No school

Attended some school

Completed primary education

Completed secondary education

Completed tertiary education

Read and write, no school

Father's education, \% of reporting (n)

No school

Attended some school

Completed primary education

Completed secondary education

Completed tertiary education

$10.1 \%(63)$

$19.1 \%(119)$

$20.4 \%(127)$

$21.8 \%(136)$

$19.9 \%(124)$

$8.8 \%(55)$

$27.1 \%(168)$

$23.4 \%(145)$

$22.7 \%(141)$

$12.4 \%(77)$

$10.8 \%(67)$

$3.5 \%(22)$

$14.7 \%(89)$

$17.1 \%(103)$

$23.0 \%(139)$

$23.5 \%(142)$

$19.0 \%(115)$

Read and write, no school

$2.6 \%(16)$

Child lives with, \% reporting (n)

Mother and father

$28.2 \%(176)$

Mother only

$35.7 \%(223)$

Father only

Other relative

$12.2 \%(76)$

$19.1 \%(119)$

Other

$4.8 \%(30)$

Parents alive, \% both alive

$48.0 \%(299)$

Caregiver HIV positive, \% yes

$71.1 \%(443)$

Caregiver has HIV healthcare, \% yes

$71.3 \%(440)$

Caregiver on ART, \% yes

$69.7 \%(434)$

Other siblings with HIV, \% yes

$15.0 \%(94)$

Other siblings who died of HIV, \% yes

$9.4 \%(59)$

${ }^{*}$ Not all percentages reflect a total of 628 participants as some with missing or unknown values.

ART, anti-retroviral treatment.

WHO. ${ }^{4}$ On the other hand, recent WHO guidelines also recommended the use of clinical and immunological parameters to target the use of viral load testing for treatment monitoring and diagnosis of first-line ARTF in settings where access to viral load is scarce. ${ }^{16}$ In most resource-limited settings and in communities living in 
Table 2 Clinical characteristics of the cohort of HIV-positive children at enrolment

\begin{tabular}{ll}
\hline Height, z-score, mean (SD) & $-1.02(1.45)$ \\
BMI, z-score, mean (SD) & $-0.90(1.26)$ \\
\% malnourished (BMI z-score <2) & $17.4(100)$ \\
$\%$ adequate nourishment (-2>BMI & $81.4(468)$ \\
Z-score <2) & \\
\% obese (BMI z-score >2) & $1.2(7)$ \\
Child took ARV prophylaxis after birth, & $1.1 \%(7)$ \\
\% yes (n) & \\
Clinical stage at initiation of ART, \% of total (n) \\
Stage 1 & $20.6 \%(129)$ \\
Stage 2 & $35.1 \%(220)$ \\
Stage 3 & $37.1 \%(232)$ \\
Stage 4 & $7.2 \%(45)$ \\
Clinical stage at enrolment, \% of total (n) & \\
Stage 1 & $88.6 \%(552)$ \\
Stage 2 & $6.9 \%(43)$ \\
Stage 3 & $4.0 \%(25)$ \\
Stage 4 & $0.5 \%(3)$ \\
Viral load at initiation, \% yes (n) & $0.8 \%(5)$ \\
Substitution of ART drugs, \% yes (n) & $42.6 \%(253)$ \\
Reason for substitution, \% of those with substitution (n) \\
Toxicity or side effects & $14.7 \%(39)$ \\
Tuberculosis coinfection & $0.8 \%(2)$ \\
National Guideline Change & $70.3 \%(187)$ \\
Total ART duration, months, mean (SD) & $45.4(35.8)$ \\
ART regimen, \% of total (n) & \\
AZT, 3TC, EFV & $13.5 \%(84)$ \\
AZT, 3TC, NVP & $45.9 \%(286)$ \\
D4T, 3TC, EFV & $5.5 \%(34)$ \\
D4T, 3TC, NVP & $20.4 \%(127)$ \\
TDF, 3TC, EFV/NVP & $9.6 \%(60)$ \\
AZT,D4T, 3TC, PI & $0.3 \%(2)$ \\
ABC, 3TC, NVP/EFV/PI & $4.8 \%(30)$ \\
Adherence of caregiver if taking ART over & $93.7 \%(12.3)$ \\
past month by VAS, mean (SD) & \\
Adherence of child reported by caregiver & $94.4 \%(11.9)$ \\
over past month by VAS, mean (SD) & \\
Tuberculosis, \% ever diagnosed (n) & $24.7 \%(154)$ \\
Type of TB diagnosed, \% with ever TB & \\
diagnosis (n) & \\
Pulmonary & $91.2 \%(134)$ \\
Disseminated & $3.4 \%(5)$ \\
Lymph node & $4.8 \%(7)$ \\
Currently prescribed PCP prophylaxis, & $32.6 \%(202)$ \\
$\%$ yes (n) & \\
Caregiver worried about stigma, \% yes (n) & $23.9 \%(150)$ \\
Child's HIV status disclosed to them, & $45.6 \%(283)$ \\
\% yes (n) & \\
\hline ART, anti-retroviral treatment; ARV, anti-retroviruses; BMI, body \\
mass index; PCP, pneumocystis jirovecii; TB, tuberculosis.
\end{tabular}

remote places, the use of viral load testing as a point-of-care monitoring and diagnostic tool is difficult given the technical requirements of the procedure, the need for an efficient sample transport system and the limited availability of the required reagents as is the case with the regional laboratory where the current cohort is located.
Table 3 Baseline laboratory characteristics at enrolment of the cohort of HIV-positive children

CD4 count at baseline, median (IQR)

741 (517-1014)

CD4 count, \% above treatment failure threshold by age (n)

Less than 5 years old, CD4 $\geq 200, \quad 100$ (32)

$\%$ of tested $(\mathrm{n})$

5 years or older, CD4 $\geq 100$,

$\%$ of tested (n)

WCC, median (IQR)

$96.9(558)$

Total lymphocyte count, median (IQR)

$6.3(4.9-8.0)$

Haemoglobin, median (IQR)

Platelet count, median (IQR)

Urinalysis, \% normal (n)

BUN, median (IQR)

$\mathrm{Cr}$, median (IQR)

SGOT, median (IQR)

SGPT, median (IQR)

$3.4(2.3-36.1)$

$13.4(12.4-14.6)$

$314(253-377)$

$95.7 \%(517)$

$7.5(5-11.5)$

$0.52(0.40-0.70)$

$32(25-39)$

$22(16-31)$

BUN, blood urea nitrogen; $\mathrm{Cr}$, creatinine; SGOT, serum glutamic oxaloacetic transaminase; SGPT, serum glutamic pyruvic transaminase; WCC, white cell count.

Performance of the WHO guidelines for detection of first-line ARTF in children has been inadequately evaluated so far, and no local scoring system has been developed for use in HIV-infected children who are on first-line ART. Children are assumed to have different predictors of treatment failure because of the differences in mode of transmission, faster disease progression and previous exposure to ART for purpose of PMTCT. ${ }^{25}{ }^{26}$ Analysis of the predictors of first-line ARTF and assessing the level of accurateness of the WHO criteria is of paramount importance for the development of such tools.

To the best of our knowledge, this is the largest prospective, paediatric HIV cohort in Africa designed to identify a prediction rule using a composite of clinical and immunological parameters to prioritise viral load testing for children on first-line ART. Its prospective design allows for better data completeness and quality. However, challenges to the current cohort are multifaceted, including poor adherence to and defaulting from care and treatment. Although we have devised strategies, including better counselling and tracking systems to decrease the defaulter rate, it still remains to be a challenge because of the large geographic area served by each ART centre. Additionally, for information to be collected retrospectively including baseline laboratory values, clinical data and previous adherence, incomplete records pose a threat to data completeness and validity. The issue of adherence is particularly significant as changing to a second-line regimen due to poor adherence would likely not improve mortality and would potentially have consequences for emergence of resistant strains. While our method of using VAS for adherence has been validated in multiple settings, it has been found to overestimate adherence when compared with other methods such as automated assessment of bottle opening. ${ }^{27}$ One potential adjunct to this study would be 
resistance testing of individual viral samples to assess resistance versus non-adherence though this is currently not part of the study protocol due to funding and resource constraints.

A prediction rule to be developed will be used in resource poor settings, especially remote areas with limited access to viral load testing for prioritisation and treatment decision purposes. It will potentially decrease the HIV-related mortality in children who would otherwise remain on a failing regimen because of lack of access to viral load testing. Additionally, it will help to investigate the effectiveness of ART in children. The potential of the prediction rule for overdiagnosis of ARTF and unnecessary switching will also be examined.

Acknowledgements The authors thank Hawassa University for supporting the project. They also acknowledge all the data collectors, children and parents for taking their time to be involved in the research.

Contributors BTT conceived the idea. BTT and BAF developed the letter of intent (LOI). BTT, BAF, DJ and AR all contributed with the full proposal development, writing, analysis and editing of the manuscript.

Funding This project is supported by International AIDS Society, CIPHER Paediatric HIV Research Grant Programme (grant ID: 307-TAD).

Competing interests None declared.

Provenance and peer review Not commissioned; externally peer reviewed.

Data sharing statement Our data can be accessed on a case-by-case basis after IRB approval is secured from Hawassa University.

Open Access This is an Open Access article distributed in accordance with the Creative Commons Attribution Non Commercial (CC BY-NC 4.0) license, which permits others to distribute, remix, adapt, build upon this work noncommercially, and license their derivative works on different terms, provided the original work is properly cited and the use is non-commercial. See: http:// creativecommons.org/licenses/by-nc/4.0/

\section{REFERENCES}

1. UNAIDS. AIDSInfo 2015. http://aidsinfo.unaids.org/ (accessed 3 Jul 2016).

2. Musoke PM, Mudiope P, Barlow-Mosha LN, et al. Growth, immune and viral responses in HIV infected African children receiving highly active antiretroviral therapy: a prospective cohort study. BMC Pediatr 2010;10:56.

3. Verweel G, van Rossum AM, Hartwig NG, et al. Treatment with highly active antiretroviral therapy in human immunodeficiency virus type 1-infected children is associated with a sustained effect on growth. Pediatrics 2002;109:E25.

4. WHO. Consolidated guidelines for the use of antiretroviral drugs for treating and preventing HIV Infection. Recommendations for public health approach. Geneva: WHO, 2013. http://appswhoint/iris/ bitstream/10665/85321/1/9789241505727 engpdf?ua=1

5. Bacha T, Tilahun B, Worku A. Predictors of treatment failure and time to detection and switching in HIV-infected Ethiopian children receiving first line anti-retroviral therapy. BMC Infect Dis 2012;12:1-8.

6. Workneh N, Girma T, Woldie M. Immunologic and clinical outcomes of children on HAART: a Retrospective cohort analysis at Jimma University specialized hospital. Ethiop $J$ Health $S c i$ 2009;19:2.

7. Peacock-Villada E, Richardson BA, John-Stewart GC. Post-HAART outcomes in pediatric populations: comparison of resource-limited and developed countries. Pediatrics 2011;127:e423-41.

8. Ayalew MB, Kumilachew D, Belay A, et al. First-line antiretroviral treatment failure and associated factors in HIV patients at the University of Gondar Teaching Hospital, Gondar, Northwest Ethiopia HIV/AIDS (Auckland, NZ) 2016;8:141-6.

9. Teshome W, Tefera A. Detection of immunological treatment failure among HIV infected patients in Ethiopia: a retrospective cohort study. BMC Immunol 2015;16:55.

10. Teshome Yimer Y, Yalew AW. Magnitude and predictors of anti-retroviral treatment (ART) failure in private health facilities in Addis Ababa, Ethiopia. PLoS One 2015;10:e0126026.

11. Hammer SM, Saag MS, Schechter M, et al. Treatment for adult HIV infection: 2006 recommendations of the International AIDS Society--USA panel. Top HIV Med2006;14:827-43.

12. Fiscus SA, Cheng B, Crowe SM, et al. HIV-1 viral load assays for resource-limited settings. PLoS Med 2006;3:e417.

13. Calmy A, Ford N, Hirschel B, et al. HIV viral load monitoring in resource-limited regions: optional or necessary? Clin Infect Dis 2007:44:128-34.

14. Schooley RT. Viral load testing in resource-limited settings. Clin Infect Dis 2007;44:139-40.

15. UNAIDS. 90-90-90 An ambitious treatment target to help end the AIDS epidemic. 2014. http://wwwunaidsorg/sites/default/files/media asset/90-90-90_en_Opdf (accessed 20 Jun 2016).

16. WHO. Consolidated guidelines on the use of antiretroviral drugs for treating and preventing HIV infection: recommendations for a public health approach—second edition. 2016. http://wwwwhoint/hiv/pub/ arv/arv-2016/en/ (accessed 20 Jun 2016)

17. WHO. Antiretroviral therapy for HIV infection in infants and children: towards universal access. Recommendations for Public Health Approach. Geneva: WHO, 2010. http://whqlibdocwhoint/publications/ 2010/9789241599801_engpdf?ua=1

18. Institute EHaNR. HIV related estimates and projections for Ethiopia-2012. Addis Ababa, Ethiopia. 2012. http://wwwunaidsorg/ sites/default/files/en/media/unaids/contentassets/documents/ data-and-analysis/tools/spectrum/Ethiopia2012reportpdf

19. WHO. Child growth standards. Switzerland, Geneva. http:// wwwwhoint/childgrowth/standards/en/

20. Amico KR, Fisher WA, Cornman DH, et al. Visual analog scale of ART adherence: association with 3-day self-report and adherence barriers. J Acquir Immune Defic Syndr 2006:42:455-9.

21. Seymour DG, Green M, Vaz FG. Making better decisions: construction of clinical scoring systems by the SpiegelhalterKnill-Jones approach. BMJ 1990;300:223-6.

22. Lynen L, An S, Koole O, et al. An algorithm to optimize viral load testing in HIV-positive patients with suspected first-line antiretroviral therapy failure in Cambodia. J Acquir Immune Defic Syndr 2009;52:40-8.

23. Westley BP, DeLong AK, Tray CS, et al. Prediction of treatment failure using 2010 World Health Organization Guidelines is associated with high misclassification rates and drug resistance among HIV-infected Cambodian children. Clin Infect Dis 2012;55:432-40.

24. Vaz P, Augusto O, Bila D, et al. Surveillance of HIV drug resistance in children receiving antiretroviral therapy: a pilot study of the World Health Organization's generic protocol in Maputo, Mozambique. Clin Infect Dis 2012;54(Suppl 4):S369-74.

25. Greig JE, du Cros PA, Mills C, et al. Predictors of raised viral load during antiretroviral therapy in patients with and without prior antiretroviral use: a cross-sectional study. PLoS One 2013;8:e71407.

26. Kellerman SE, Ahmed S, Feeley-Summerl T, et al. Beyond prevention of mother-to-child transmission: keeping HIV-exposed and HIV-positive children healthy and alive. AIDS 2013;27(Suppl 2): S225-33.

27. Muller $A D$, Jaspan HB, Myer L, et al. Standard measures are inadequate to monitor pediatric adherence in a resource-limited setting. AIDS Behav 2011;15:422-31. 